\title{
PROSPECTS FOR THE USE OF HIGH TEMPERATURE SUPERCONDUCTORS IN HIGH FIELD ACCELERATOR MAGNETS"
}

\author{
D. C. Larbalestier ${ }^{+}$and P. J. Lee, \\ Applied Superconductivity Center, University of Wisconsin-Madison, Madison WI
}

\begin{abstract}
Several future accelerators will require magnets to operate above $10 \mathrm{~T}$ (for example the proposed Muon and Hadron colliders). In this new domain of accelerator magnets, the pre-eminence of $\mathrm{Nb}-\mathrm{Ti}$ falls away. In the time frame of new accelerator construction (10-20 years), there are strong opportunities to bring on new classes of superconductor (advanced A15, HTS), provided that serious, focused efforts start soon. Of the primary HTS superconductors, $\mathrm{Bi}_{2} \mathrm{Sr}_{2} \mathrm{CaCu}_{2} \mathrm{O}_{x}, \quad(\mathrm{Bi}, \mathrm{Pb})_{2} \mathrm{Sr}_{2} \mathrm{Ca}_{2} \mathrm{Cu}_{3} \mathrm{O}_{x}, \quad$ and $\mathrm{YBa}_{2} \mathrm{Cu}_{3} \mathrm{O}_{7}$, the most promising for near-term high field magnet application is $\mathrm{Bi}_{2} \mathrm{Sr}_{2} \mathrm{CaCu}_{2} \mathrm{O}_{x}(\mathrm{Bi}-2212)$. However, HTS conductors are still at an early stage of development and continued improvement over the next ten years could make other HTS superconductors available for accelerator application. Bi-2212 appears to have the highest potential today, because it can be made in round wire form with reasonably high $J_{\mathrm{c}}$ values, thus permitting access to the cabling technology developed for LTS materials. Bi-2223 and YBCO are both presently limited to wide-tape designs, for which cabling is a significant challenge. Development of less aspected conductor designs might permit YBCO coated conductors to drive out 2212 as the present conductor of choice. An alternative approach is to design magnets around the use of aspected conductor forms and anisotropic properties in order to make the most of the unique properties of HTS superconductors.
\end{abstract}

\section{INTRODUCTION}

It has been 13 years since the discovery of high temperature superconductors (HTS) [1] but it is likely to be at least as long until a next generation of accelerators that might fully exploit them goes into construction. Some planning and speculation as to how HTS might enter into HEP construction plans is therefore appropriate. At this time, conductors made from HTS have made considerable progress towards viability for magnets useful for utility applications, where the essential need is that HTS replace copper and iron. This means that their main present target is dominated by lower field uses (1-2 T, occasionally higher) than most LTS applications, and by cost. Today, HTS conductors made of multifilamentary Bi-2223

\footnotetext{
*Work supported by the US Dept. of Energy, Division of High Energy Physics grant DE-FG02-91ER40643.

\# Email: larbales@engr.wisc.edu, lee@engr.wisc.edu

+Also the Department of Materials Science and Engineering and the

Department of Physics at the University of Wisconsin-Madison.
}

$\left((\mathrm{Bi}, \mathrm{Pb})_{2} \mathrm{Sr}_{2} \mathrm{Ca}_{2} \mathrm{Cu}_{3} \mathrm{O}_{\mathrm{x}}\right)$ (see Figure 1) are being applied to significant prototype motors, transformers, power cables, fault current limiters, and other utility applications [2]. The principal limitation on their eventual use is now seen to be primarily economic, since most electrotechnical and utility applications are already available from conventional uses of $\mathrm{Cu}, \mathrm{Fe}$ and $\mathrm{Al}$. Accelerator applications are generally fundamentally different, in that superconductivity is a vital enabling technology and they are also demanding in what they expect of the superconductor. Thus the HEP view of HTS is not at all the same as the utility market view of HTS

\section{HEP MAGNET SYSTEMS}

There are essentially two types of magnet that HEP wants, dipole and quadrupole magnets with bores of a few tens of millimeters for beam steering, and large detector magnets of many meters diameter for beam interaction analysis. Present designs of large hadron colliders (e.g. LHC) are already at $8 \mathrm{~T}$ for main ring dipoles and more than $10 \mathrm{~T}$ for specialty quadrupoles. By contrast, beam interaction analysis magnets are often meters in diameter and they continue to get bigger. However, their fields remain in the 1-2 $\mathrm{T}$ range, which is comfortably accessible by present day $\mathrm{Nb}$-Ti conductors.

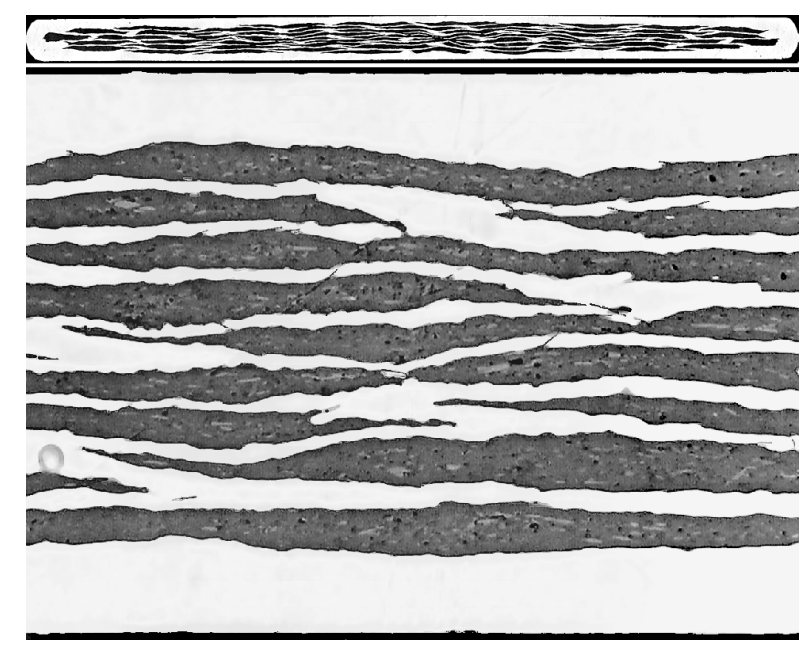

Figure 1: Cross-sectional detail of 85 Filament $(\mathrm{Bi}, \mathrm{Pb})_{2} \mathrm{Sr}_{2} \mathrm{Ca}_{2} \mathrm{Cu}_{3} \mathrm{O}_{x}$ conductor from American Superconductor Corporation with inset overview of cross-section. Typical dimensions of such a conductor are $4 \mathrm{~mm}$ by $0.2 \mathrm{~mm}$ and the zero field critical current is about $100 \mathrm{~A}$ at $77 \mathrm{~K}$ and $500 \mathrm{~A}$ at $4.2 \mathrm{~K}$. 




Figure 2: Y-123 coated conductors in the two presently favored formats. The upper cross-section of a rollingassisted-biaxially-textured-substrate (RABiTS ${ }^{\mathrm{TM}}$ ) conductor made at ORNL and imaged by Yang and Babcock at UW shows the complex substrate ([001]-textured Ni), 3-layer buffer of $\mathrm{CeO}_{2} / \mathrm{Y}_{2} \mathrm{O}_{3}$-stabilized $\mathrm{ZrO}_{2}$ (YSZ) with superconducting YBCO overlayer. The alternate approach developed by Fujikura Electric and LANL is illustrated at bottom (courtesy S. Foltyn at LANL). In this case the substrate is untextured and the buffer layer (normally YSZ) is textured by ion-beam-assisted-deposition (IBAD) process. Both routes lead to a wide-tape, monofilament conductor. Typical dimensions are $1 \mathrm{~cm}$ wide, $1-2 \mu \mathrm{m}$ thick Y-123, $1 \mu \mathrm{m}$ thick buffer layer(s) and $100 \mu \mathrm{m}$ thick substrate. Such a conductor can have a critical current of 100-200 A at $77 \mathrm{~K}$ and some $7-10$ times larger at $4 \mathrm{~K}$.

\section{HTS CONDUCTOR OPTIONS}

Today there are three HTS materials from which useful conductors can be made. They are the two micaceous $\mathrm{Bi}$ Sr-Ca-Cu-O (BSCCO) compounds $\mathrm{Bi}_{2} \mathrm{Sr}_{2} \mathrm{CaCu}_{2} \mathrm{O}_{x}$ (Bi$2212)$ with $T_{\mathrm{c}} \sim 90 \mathrm{~K}$ and $(\mathrm{Bi}, \mathrm{Pb})_{2} \mathrm{Sr}_{2} \mathrm{Ca}_{2} \mathrm{Cu}_{3} \mathrm{O}_{\mathrm{x}}(\mathrm{Bi}-2223)$ with $T_{\mathrm{c}} \sim 107 \mathrm{~K}$, and the metallic reservoir layer compound $\mathrm{YBa}_{2} \mathrm{Cu}_{3} \mathrm{O}_{7-\mathrm{x}}(\mathrm{Y}-123)$ with $T_{\mathrm{c}}$ of $\sim 92 \mathrm{~K}$. However, in the context of a still great worldwide interest in high temperature superconductivity (and the very recent report of surface superconductivity [3] in the Na-doped $\mathrm{WO}_{3}$ ), we should still admit the possibility of the discovery of new compounds that might replace the present choices. Most of us in the superconductivity community have a mindset that postulates that new superconductors of higher $T_{\mathrm{c}}$ are going to be more complex than any low temperature superconductor (LTS). However, there is no firm and explicit basis for his belief, and we should remain open to pleasant surprises. Those who are optimists may be heartened by the recent interest of the popular author, Tom Clancy, in superconductivity. In his 1999 book, "Carrier", he describes the discovery of superconductivity well above RT in wires of a Cu-Pt-Sc mix [4] in the year 2016! Apparently the simultaneous discovery of large Pt and Sc reserves in Sri Lanka also makes the material very affordable, halts a nuclear skirmish between India and Pakistan, and leads to the award of both the Physics and Chemistry Nobel prize to the discoverer and to instant widespread applications too!

Turning back to today's conductor designs, they are either large aspect ratio multifilaments (Figure 1), round wire multifilaments (Figure 3) or monofilament tapes (Figure 2), Bi-2223 (or Bi-2212) exemplifying the first, $\mathrm{Bi}-2212$ the second, and Y-123 the third. Before going into the details of fabrication that govern their availability, we first turn to discussion of the underlying properties that determine their suitability for conductors. The key issues that must be addressed in order for HTS to be made into successful conductors are:

High critical current density.

Temperature capability.

Strength.

Length availability.

Cost and performance competitiveness with LTS conductors.

Of the above issues, only improved temperature capability by HTS conductors is fully satisfied in the competition between HTS and LTS superconductors such as Nb-Ti and $\mathrm{Nb}_{3} \mathrm{Sn}$. Attaining high enough overall $J_{\mathrm{c}}$ in reasonable conductor forms is the biggest present obstacle to applications and the one that we emphasize most in the limited space available here.

\section{PREREQUISITES FOR HTS USE}

\subsection{High Critical Current Density}

The most fundamental requirement of any viable conductor is that it must have a high critical current density, $J_{c}$, in the field range needed for the magnet. High normally is taken to mean values of $>10^{3} \mathrm{~A} / \mathrm{mm}^{2}$ flowing in the superconductor cross-section. For low temperature superconductors, we expect that $J_{\mathrm{c}}$ is primarily determined by flux pinning (filament cross-sectional variation (sausaging) may reduce the flux pinning determined $J_{c}$ by factors of order $10 \%$ ), but for HTS materials this is very far from being the case. Figure 4 shows that the best HTS conductors exceed this baseline value, BSCCO at $4 \mathrm{~K}$ (and perhaps up to $15-20 \mathrm{~K}$ ), while Y-123 does this comfortably at $77 \mathrm{~K}$. However, a central fact of all HTS polycrystalline forms is that the supercurrent percolates, because it is im- 


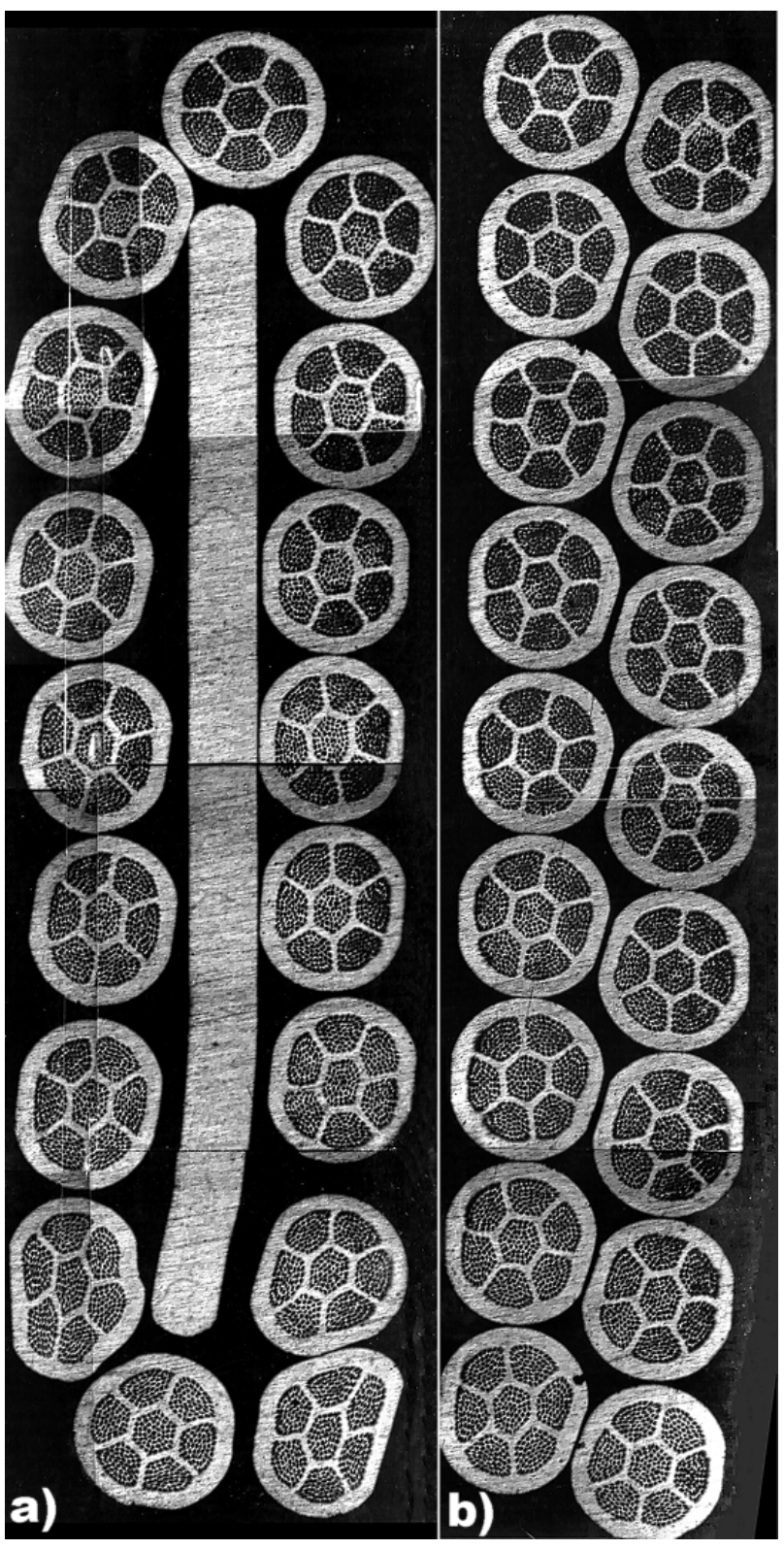

Figure 3: Cable wound at LBNL from round multifilamentary "double stack" Bi-2212 conductor made by Showa Electric a) with and b) without Ag core. The $I_{c}$ values are around $280 \mathrm{~A}$ at $4.2 \mathrm{~K}$ and self field, for the 0.8 $\mathrm{mm}$ diameter strands. Images courtesy of D. Dietderich (LBNL).

peded by obstacles on multiple length scales. This percolation results in loss of vital information in understanding the attainable current densities in many conductor forms, because the presence of so many barriers means that the actual cross-section occupied by transport current is a continuously varying and in general unknown quantity. What can be measured to high accuracy is the critical current, $I_{\mathrm{c}}$, but the conversion to $J_{\mathrm{c}}$ produced by dividing by the total cross-sectional area of superconductor contains little fundamental information.

This percolation is illustrated by representative flux penetration pictures obtained by magneto optical (MO)

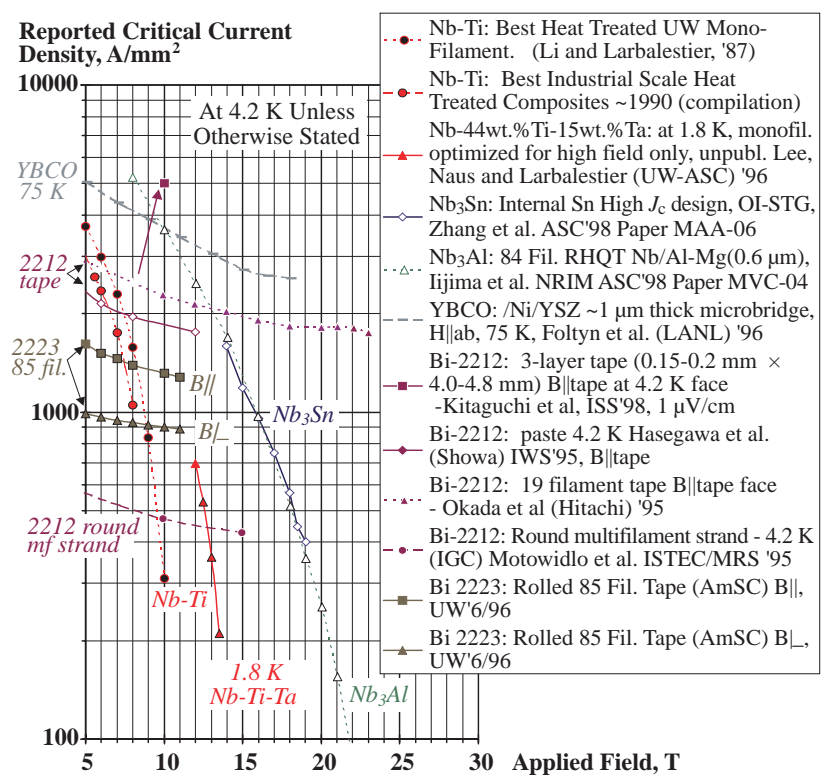

imaging of 2212, 2223, and Y-123 conductors in Figure 5 and Figure 6. The image contrast comes from the nonuniformity of flux penetrating into the superconductor. Flux obviously penetrates $\mathrm{Bi}-2212$ and $\mathrm{Bi}-2223$ differently. The fine network in Bi-2223 has its origin in the many cracks that populate 2223 filaments. Bi-2212, being melt processed at its final stage, suffers from porosity on a larger scale, while the origin of percolation in Y-123 is less clear at the present time. The larger length scale of the granularity is important, since it means that typical $\mathrm{Bi}$ 2212 conductors have overall $J_{c}$ values 2-3 times those of Bi-2223 conductors at low temperatures, even though Bi2212 has the lower $T_{c}$.

At the smallest scale, the ultimate limit to $J_{c}$ is defined by the depairing current density, $J_{\mathrm{d}}$, the current density of the Meissner sheath or the circulating current around each vortex. Since $J_{\mathrm{d}} \sim 0.5 H_{\mathrm{c}} / \lambda$, where $H_{\mathrm{c}}$ is the thermodynamic critical field and $\lambda$ is the penetration depth over which currents circulate, values of $J_{\mathrm{d}}$ well exceed the needed value of $J_{c}$, since they reach $10^{6} \mathrm{~A} / \mathrm{mm}^{2}$ at $4 \mathrm{~K}$ and $10^{5} \mathrm{~A} / \mathrm{mm}^{2}$ at $77 \mathrm{~K}$. The flux pinning current density, $J_{\mathrm{fp}}$, is typically up to $10 \%$ of $J_{\mathrm{d}}$. It is determined by the density of pinning sites in the microstructure and the vortex density gradient that they can support. Thus more than adequate $J_{\mathrm{c}}$ is available from flux pinning too, even if the value of order $10^{4} \mathrm{~A} / \mathrm{mm}^{2}$ at $77 \mathrm{~K}$ must be derated somewhat to take account of flux creep, a phenomenon not normally considered in LTS materials.

The first barrier seen by these large values of local, intragranular critical current density, $J_{\text {fp }}$, are grain boundaries of arbitrary misorientation, which in general have significant local strain and disorder and depressed superconductivity, which makes the boundary a barrier to current flow. Although the magnitude of this effect is known well for special [001] tilt boundaries in Y-123 (the inter- 

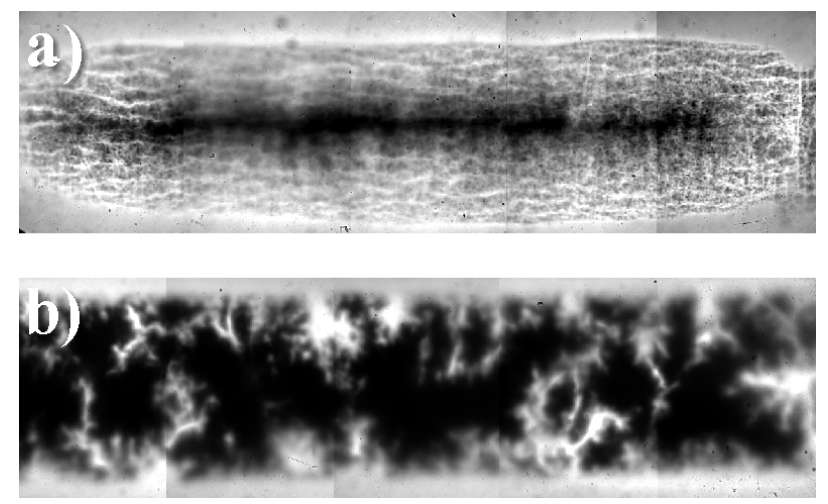

Figure 5: Magneto optical images $(\mathrm{T}=20 \mathrm{~K}, \mathrm{~B}=72 \mathrm{mT})$ of a) Bi-2223 and b) Bi-2212 tape conductors. Residual, preferentially oriented crack-like defects extend along the tape axis in the $\mathrm{Bi}-2223$ conductor as a result of thermomechanical processing. The Bi-2212 exhibits better flux shielding because it does not undergo a heavy mechanical deformation step after heat treatment. However, the melting step inherent in its optimum processing produces larger scale defects such as pores and secondary phases. The generally better connectivity of $\mathrm{Bi}-2212$ means that its low temperature critical current density is higher than that of $\mathrm{Bi}-2223$, even though $\mathrm{Bi}-2223$ has the higher transition temperature. Images courtesy of A. A. Polyanskii (UW).

granular $J_{c}, J_{g b}$, is depressed by about $10^{3}[5,6]$ on increasing the misorientation from 0 to $45^{\circ}$ ), it is not well known for most boundaries and there are interesting signs that the doping state of the compound plays an important role in determining and perhaps ameliorating these strong barriers to current flow [7]. This strong dependence on misorientation is the reason for using texturing methods for making Y-123 coated conductors, as noted in Figure 2.

Larger scale barriers are cracks and voids, which are in these authors opinions [8-9] amongst the most serious contributors to the degradation of $J_{\mathrm{c}}$ in the Bi compounds, while sausaging also contributes 20 to $100 \%$ degradations to $J_{\mathrm{c}}$. Thus the final $J_{\mathrm{c}}$ determined by the measurements of $I_{\mathrm{c}}$ and $A_{\text {total }}$ is a much reduced, trickledown $J_{\mathrm{c}}$, in which an inherently high flux pinning $J_{\mathrm{c}}$ is reduced by factors of order 10 to 100 in BSCCO and perhaps 5-10 in Y-123. This is of course also a big opportunity to improve the $J_{c}$ since the factors controlling $J_{\mathrm{c}}$ are not fundamental ones, but rather those affected by the processing into useful conductor forms.

We finally should note that the working $J_{c}$ of the conductor is further diluted by the support structure (as seen in Figure 2, the superconductor cross-section in coated conductors is only about $1 \%$ of the substrate and buffer layer) and the need to add a normal metal stabilizer, typically about equal to the superconductor cross-section. Thus the $J_{\mathrm{c}}$ values of Figure 4 may be seriously misleading when applied to real conductors, of which in any case only BSCCO are available in lengths beyond $1 \mathrm{~m}$ today.

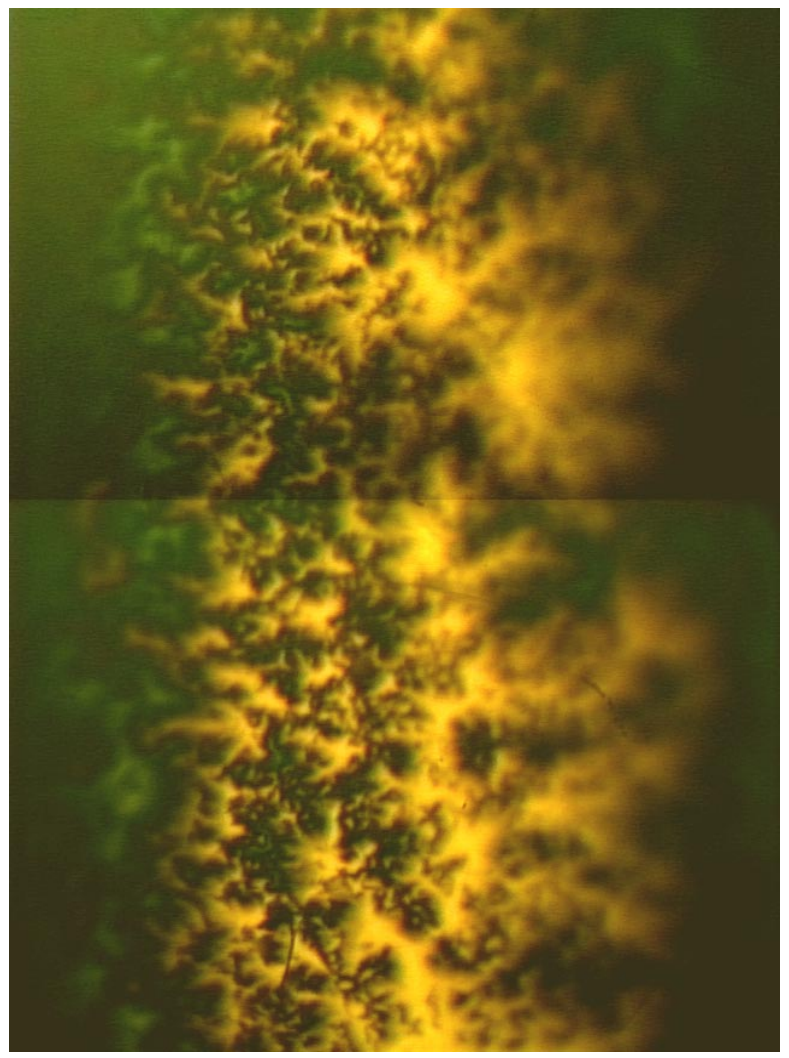

Figure 6: Magneto Optical image (FC in $60 \mathrm{mT}$ to $77 \mathrm{~K}$, field then decreased to zero) of a $0.6 \mathrm{MA} / \mathrm{cm}^{2}(77 \mathrm{~K}, 0 \mathrm{~T})$ Y-123 coated conductor tape (FAL5727) showing the granular flux penetration characteristic of supercurrent percolation. Sample courtesy of A. Goyal (ORNL and image courtesy of A. Pashitski (UW).

\subsection{Temperature Capability}

As suggested by Figure 4, it seems that only Y-123 is viable for making strong magnets at $77 \mathrm{~K}$. The determining factor is the irreversibility field $H^{*}(\mathrm{~T})$ at which the $J_{\mathrm{c}}$ goes to zero [10]. This has typical values of $5 \mathrm{~T}$ (Y-123) and $0.3 \mathrm{~T}$ (Bi-2223) at $77 \mathrm{~K}$. The BSCCO compounds do not achieve $H^{*}$ which exceeds $5 \mathrm{~T}$ until the temperature is reduced below about $25-30 \mathrm{~K}$, while Y-123 has $H^{*}$ exceeding $20 \mathrm{~T}$ at such temperatures.

\subsection{Strength}

Pure Ag is very soft, but it can be hardened by alloying additions. Ag doped with $\sim 0.5-2$ wt. $\% \mathrm{Mg}$ is the most favored present alloy, permitting yield strengths up to $350-\mathrm{Mpa}$ which is more than 5 times the value for $\mathrm{Ag}$ sheathed tapes [11].

\subsection{Length Availability}

Today BSCCO-2223 available in 0.5-1 km lengths from several companies world wide in conductor forms such as that shown in Figure 1. Since such wide tape conductors have $I_{\mathrm{c}}$ values of $100 \mathrm{~A}$ or less, they often need to be assembled in parallel. For magnets co-winding a stack of 3-5 
conductors is presently the most favored method, but this is rather primitive compared to accelerator demands and expectations from LTS conductors, where the fully transposed Rutherford cable is generally favored. Full (or at least one-layer) transposition can be attained in power cables by twisting the tapes around a core, since only operation in self field is required and the loss of space (often used for cooling) in the core is not material. The Pipatron concept for making a VLHC magnet is compatible with this form of cable [12]. Since the need to make aspected conductors from HTS compounds is fundamentally driven by their layered atomic structure, there is an important need to find new ways to cable HTS conductors or to use aspected conductors in new magnet designs. Recent common coil designs may be one such approach [13].

The most promising present conductor for saddle coil applications is in fact $\mathrm{Bi}-2212$. The melt processing that is applied to make 2212 permits reasonable connectivity (the main factor controlling percolation and thus $J_{\mathrm{c}}$ ) in round wire form. Scanlan's group at LBNL have been developing this with several manufacturers and one such cable operating at $\sim 4000 \mathrm{~A}$ at $4 \mathrm{~K}$ is shown in Figure 3. Such a cable is now being made in lengths of $\sim 100 \mathrm{~m}$ and will soon be tested in coil designs common to $\mathrm{Nb}-\mathrm{Ti}$ and $\mathrm{Nb}_{3} \mathrm{Sn}$ conductors too, permitting a direct comparison of HTS and LTS performance.

Since Y-123 conductors are not yet available in more than $1 \mathrm{~m}$ lengths and their form is that shown in Figure 2, they face several problems common to Bi-2223, exacerbated for now at least by the very large fraction of substrate that so strongly reduces the overall $J_{\mathrm{c}}$ of the conductor. Much present effort world-wide is aimed at reducing this overall $J_{c}$ limitation and in scaling up for longlength manufacture.

\subsection{Costs}

Present costs of HTS wire are high but since they are made on a still small scale with much high-priced characterization, it seems not necessary to be too strongly concerned by present costs for the long term. The raw material costs of $\mathrm{Ag}$ and $\mathrm{BSCCO}$ powder are within about a factor of two of the same for $\mathrm{Cu}$ and $\mathrm{Nb}$ - $\mathrm{Ti}$, but conductor costs expressed in $\$ / \mathrm{kA} . \mathrm{m}$ are more than a factor of 100 different. Thus raising $J_{c}$ and scale up are crucial parameters to be addressed for HTS conductors.

\section{CONCLUSIONS}

As this very brief review has summarized, HTS conductors suitable for HEP saddle coil applications are still some way from being commodity items, because they are still primitive, of lower working overall $J_{\mathrm{c}}$ and higher cost than competing LTS conductors. But LTS conductors have had some 35 years of development, strong improvements coming even in the last 10 years, making it not appropriate either to abandon LTS or HTS conductors. HEP magnet builders with HTS interests can now start to play an im- portant role in developing the technology, pushing the development of HTS conductors in ways that have been so productive for LTS conductors [14]. And although not discussed here, HTS current leads are likely to be applied to all future accelerators, providing the first entry point of the new technology to HEP.

\section{ACKNOWLEDGEMENTS}

We are grateful to colleagues in the coated conductor development group and wire development group for many discussions about HTS conductor characterisation and development and to AFOSR, DOE-EERE, EPRI, and NSF-MRSEC for support of the Wisconsin HTS programs.

\section{REFERENCES}

[1] J. G. Bednorz and K. A. Muller, "Possible high $T_{\mathrm{c}}$ superconductivity in the Ba-La-Cu-O system," Zeitschrift-fur-Physik-B, vol.64(2), pp.189-93, (1986).

[2] R. D. Blaugher, "Superconducting electric power applications," Adv. Cryo. Eng., vol. 42, pp. 883-898, (1996).

[3] S. Reich and Y. Tsabba, "Possible nucleation of a 2D superconducting phase on $\mathrm{WO}_{3}$ single crystals doped with $\mathrm{Na}^{+}$," to be published in the European Physical Journal B, 1999.

[4] Tom Clancy, "Carrier; a guided tour of an aircraft carrier," Berkley Pub., 1999.

[5] D. Dimos, P. Chaudhari, J. Mannhart, " Superconducting transport properties of grain boundaries in $\mathrm{YBa}_{2} \mathrm{Cu}_{3} \mathrm{O}_{7}$ bicrystals," PhysicalReview-B-(Condensed-Matter), vol.41(7), pp. 4038-49, (1990).

[6] N. F. Heinig, R. D. Redwing, J. E. Nordman, and D. C. Larbalestier, "The strong to weak coupling transition in low misorientation angle thin film YBa2Cu3O7-x bicrystals", to be published in Physical Review B, (1999).

[7] A. Gurevich, E. A. Pashitskii, " Enhancement of superconductivity at structural defects in high-temperature superconductors," PhysicalReview-B-(Condensed-Matter). vol.56(10), pp.6213-25, (1997).

[8] D. C. Larbalestier, "The road to conductors of high temperature superconductors: 10 years do make a difference," IEEE Transactions On Applied Superconductivity, vol. 7(2), pp. 90-97 (1997).

[9] X. Y. Cai, A. Polyanskii, Q. Li, G. N. Riley, and D. C. Larbalestier, "Current-limiting mechanisms in individual filaments extracted from superconducting tapes," Nature, vol. 392, pp. 906-909 (1998).

[10] D. Larbalestier, J. W. Anderson, S. E. Babcock, X. Y. Cai, S. E. Dorris, M. Feldmann, J. Jiang, Q. Li, J. A. Parrell, R. Parrella, M. Polak, A. Polyanskii, G. N. Riley Jr., M. Rupich, and Y. Wu. "New Experiments Elucidating the Current Limiting Mechanisms of AgSheathed (Bi,Pb)2Sr2Ca2Cu3Ox Tapes," To appear in Advances in Superconductivity XI, Springer Verlag Tokyo (1999).

[11] B. Ullmann, A. Gäbler, M Quilitz, W. Goldacker, "Transport Critical Currents of Bi(2223) Tapes at 77 K Under Mechanical Stress," IEEE Trans. Applied Superconductivity, vol. 7(2), pp. 2042-2045, (1997).

[12] G.W. Foster, V. Kashikhin, M. S. McAshan, P.O. Mazur, H. Piekarz, J.T. Volk, R. Walker, "Conductor design for the VLHC transmission line magnet," paper TUBR5 at the 1999 Particle Accelerator Conference, these proceedings., (1999).

[13] R. Gupta, "Common Coil Magnet System for VLHC," paper THP120 at the the 1999 Particle Accelerator Conference, these proceedings.

[14] D. C. Larbalestier and P. J. Lee, "New Developments in Niobium Titanium Superconductors", in proceedings of The 1995 Particle Accelerator Conference, Dallas, TX, May1, pp. 1276-1281, 1996. 